Analysis of the frequency response of a dispersive IDT/ZnO/sapphire SAW filter using effective permittivity and the coupling of modes model

This article has been downloaded from IOPscience. Please scroll down to see the full text article. 2004 J. Phys. D: Appl. Phys. 37120

(http://iopscience.iop.org/0022-3727/37/1/020)

The Table of Contents and more related content is available

Download details:

IP Address: 140.112.113.225

The article was downloaded on 23/12/2008 at 02:35

Please note that terms and conditions apply. 


\title{
Analysis of the frequency response of a dispersive IDT/ZnO/sapphire SAW filter using effective permittivity and the coupling of modes model
}

\author{
Yung-Yu Chen ${ }^{1}$, Tsung-Tsong Wu and Tsung-Tai Chou \\ Institute of Applied Mechanics, National Taiwan University, Taipei, Taiwan \\ E-mail: yychen@ndt.iam.ntu.edu.tw
}

Received 22 July 2003

Published 10 December 2003

Online at stacks.iop.org/JPhysD/37/120 (DOI: 10.1088/0022-3727/37/1/020)

\begin{abstract}
In this paper, we propose a modified coupling of modes (COM) approach, which can be used in the practical design of a layered surface acoustic wave (SAW) filter. Unlike the half-space SAW filter, frequency dependences of the COM parameters are included by using the effective permittivity approach of surface waves. Owing to the introduction of the COM model, the effects of propagation loss, electrode reflections, electrical transduction, acoustic reception, thin film loss and the distributed finger capacitance can be taken into accounts in the frequency analysis of the layered SAW filter. The frequency response of a two-port $440 \mathrm{MHz}$ IDT/ZnO/R-plane sapphire layered SAW filter is analysed and compared with the existing experimental results. Results have shown good agreement between the calculated and experimental frequency responses.
\end{abstract}

\section{Introduction}

The need for increasing the frequency of a surface acoustic wave (SAW) filter without reducing the electrode spacing into the sub-micron region has led to increasing research in the field of dispersive SAW devices. By sputtering a piezoelectric thin film on a high velocity substrate, the surface wave velocity can be increased significantly. There are experimental as well as theoretical investigations on layered SAW filters with $\mathrm{ZnO}$ on a high velocity substrate. The electromechanical coupling and dispersion characteristics of SAW on layered structures with $\mathrm{ZnO}$ on sapphire or diamond have been calculated in [1-3], and experimental results on layered SAW filters were reported in [4-7].

Compared with other models for the design and analysis of SAW devices, the coupling of modes (COM) model provides an efficient and highly flexible approach for modelling various types of electrodes, and therefore, has been used widely in modelling nondispersive SAW devices for many years [8-10]. Parameters of the COM differential equations are

1 Author to whom any correspondence should be addressed. usually obtained by measurements or by borrowing from some related theoretical modelling [11-13]. Recently, some precise numerical tools to analyse SAW properties under the periodic metallic-gratings structure, and further, calculate the COM parameters have been proposed. Endoh et al [14] adopted the hybrid finite element method and spectral domain analysis to extract COM parameters for infinite periodic gratings. Ventura et al [15] demonstrate a new effective combined finite element method and periodic Green's function formalism for the analysis of periodic SAW structures. Koskela et al [16] presented a novel phase shift method to model a periodic array of electrodes.

For a layered SAW filter, since the phase velocity of the SAW is dispersive, it has to be taken into account in the design. In the past, there have been many studies on modelling the responses of layered SAW transducers. Emanetoglu et al [17] used Green's function to simulate the frequency responses of $\mathrm{ZnO} / \mathrm{R}-\mathrm{Al}_{2} \mathrm{O}_{3}$ layered SAW filters. Smith [18] derived a dyadic Green's function to analyse the $\mathrm{ZnO} /$ diamond substrate. Green's function does not, however, help us in determining the propagation properties of SAWs propagating under metal gratings. Hachigo and Malocha [19] employed the delta 
function model to calculate the null frequency bandwidth of $\mathrm{ZnO} /$ diamond/Si layered SAW filters. Gryba et al [20] presented an analysis of a ladder SAW filter built up on a $\mathrm{ZnO} / \mathrm{GaAs}$ layered structure using COM theory without taking the dispersion effect into account. In addition, the hybrid method, like the boundary integral [21] has been developed for taking into account the electrical and mechanical perturbations caused by the periodic metal gratings on a layered substrate. However, the drawback is that it is very time consuming, especially for layered structures. In a recent paper, $\mathrm{Wu}$ and Chen [22] calculated the frequency response of a $\mathrm{ZnO} /$ diamond/Si layered SAW filter using the effective permittivity approach; however, the IDT electrode effects were not included.

In this paper, we propose a modified COM approach, which can be used in the practical design of a layered SAW filter. Unlike the half-space SAW filter, frequency dependences of the COM parameters are included by using the effective permittivity approach [22]. Owing to the introduction of the COM model, the effects of propagation loss, electrode reflections, electrical transduction, acoustic reception, thin film loss and the distributed finger capacitance can be taken into account in the frequency analysis of the layered SAW filter. The frequency response of an IDT/ZnO/R-plane sapphire layered SAW filter is analysed and compared with the existing experimental results.

\section{Effective permittivity of a layered piezoelectric medium}

Based on the formulation in [22], the dispersion characteristics of an IDT/ZnO/R-plane sapphire layered system is studied (figure 1) first. The epitaxial relationships are that the (1120) plane of $\mathrm{ZnO}$ is parallel to the surface of the (0112) sapphire substrate, and the Rayleigh wave propagates along the $c$-axis of the $\mathrm{ZnO}$ film and the $(0 \overline{1} 11)$ direction of the sapphire substrate. The related constants utilized in the calculations are adopted from those listed in [23]. Shown in figure 2 is a plot of the phase velocity dispersion and the electromechanical coupling coefficient. The thin-solid line represents the phase velocity dispersion of the 0th Rayleigh mode, the thick-solid line represents that of the 1st Rayleigh mode and the broken line the electromechanical coupling coefficient of the 0th

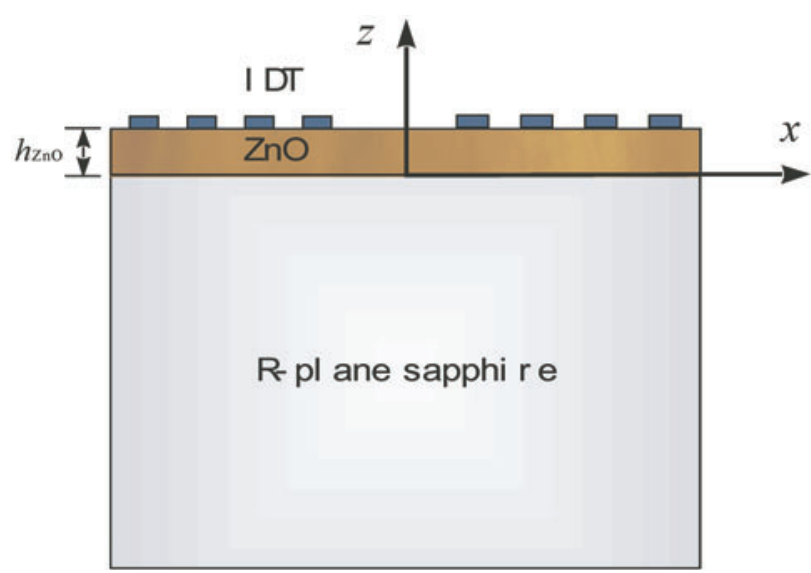

Figure 1. Coordinates of a planar-layered half space.
Rayleigh mode. The horizontal axis is the multiplication of the frequency and the thickness of the $\mathrm{ZnO}$ layer $h_{\mathrm{ZnO}}$. The result shows that the phase velocity of the 1 st Rayleigh mode is as high as $10500 \mathrm{~m} \mathrm{~s}^{-1}$ which is about three times that of conventional materials. In addition, the coupling coefficient has a maximum value of $1.3 \%$ at the frequency thickness value around $700 \mathrm{~m} \mathrm{~s}^{-1}$. In contrast to SAW in the half-space, the phase velocity and the coupling coefficient of the surface wave in the dispersive layered substrate are no longer constant; instead, they are frequency dependent.

\section{COM model for a dispersive layered SAW filter}

\subsection{COM model}

COM equations have been derived and utilized for analysing nondispersive SAW transducers with constant or arbitrary reflectivity weighting [9-11]. In the formulations, the effects of propagation loss, electrode reflections, electrical transduction, acoustic reception, thin film loss and distributed finger capacitance have been included. For a dispersive layered SAW filter, some of the COM parameters become frequency dependent due to the phase velocity dispersion and have to be modified accordingly. For a uniform transducer, the COM equations that govern the SAW mode amplitudes $R(x, \omega)$, $S(x, \omega)$ propagating in the $\pm x$ directions (figure 3 ) can be arranged in a concise form as [11]

$$
\begin{aligned}
\frac{\mathrm{d} R(x)}{\mathrm{d} x}= & -j k_{\mathrm{E}} R(x)+j K_{\mathrm{R}} \mathrm{e}^{-\mathrm{j} 2 k_{0} x} S(x)+j \alpha_{\mathrm{R}} V_{0} \mathrm{e}^{-\mathrm{j} k_{0} x}, \\
\frac{\mathrm{d} S(x)}{\mathrm{d} x}= & +j k_{\mathrm{E}} S(x)-j K_{\mathrm{S}} \mathrm{e}^{+\mathrm{j} 2 k_{0} x} R(x)-j \alpha_{\mathrm{S}} V_{0} \mathrm{e}^{+\mathrm{j} k_{0} x}, \\
\frac{\mathrm{d} I(x)}{\mathrm{d} x}= & +j 2 \alpha_{\mathrm{S}} R(x) \mathrm{e}^{+\mathrm{j} k_{0} x}+j 2 \alpha_{\mathrm{R}} S(x) \mathrm{e}^{-\mathrm{j} k_{0} x} \\
& -j\left(\frac{3 \omega C_{\mathrm{F}} / \Lambda_{\mathrm{T}}}{3+j \omega R_{\mathrm{F}} C_{\mathrm{F}}}\right) V_{0}
\end{aligned}
$$

where $\Lambda_{\mathrm{T}}$ is the wavelength of transduction, $V_{0}$ is the voltage across the IDT, $I$ is the current flow into the bus bar,

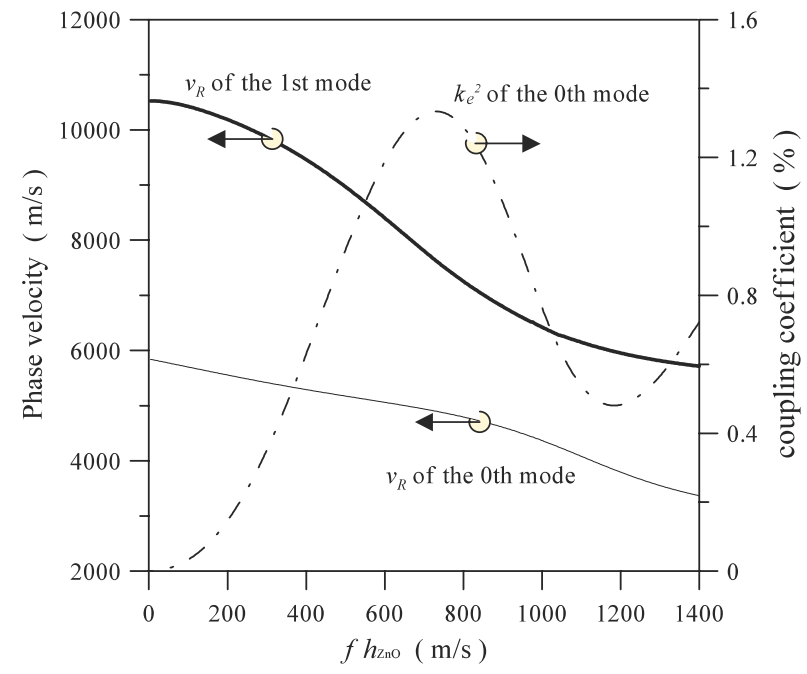

Figure 2. Electromechanical coupling coefficient and phase velocity dispersions of an IDT/ZnO/R-sapphire layered system. 


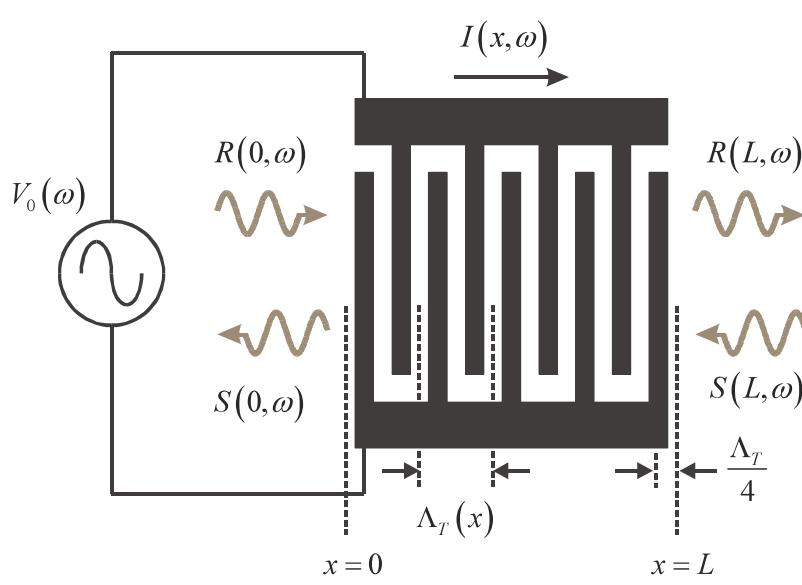

Figure 3. Coordinates of the IDT.

$k_{0}=2 \pi / \Lambda_{\mathrm{T}}$ is the transducer's synchronous wavenumber and

$$
\begin{gathered}
k_{\mathrm{E}}=+\frac{\omega}{v_{\mathrm{R}}}-\left(\frac{2 \alpha^{2} \omega C_{\mathrm{F}} R_{\mathrm{F}}^{2} \Lambda_{\mathrm{T}}}{9+\left(\omega R_{\mathrm{F}} C_{\mathrm{F}}\right)^{2}}\right) \\
-j\left(\gamma+\left(\frac{6 \alpha^{2} R_{\mathrm{F}} \Lambda_{\mathrm{T}}}{9+\left(\omega R_{\mathrm{F}} C_{\mathrm{F}}\right)^{2}}\right)\right), \\
\alpha_{\mathrm{R}}=\frac{3 \alpha \mathrm{e}^{+\mathrm{j} \phi_{\mathrm{T}}}}{3+j \omega R_{\mathrm{F}} C_{\mathrm{F}}}, \\
\alpha_{\mathrm{S}}=\frac{3 \alpha \mathrm{e}^{-\mathrm{j} \phi_{\mathrm{T}}}}{3+j \omega R_{\mathrm{F}} C_{\mathrm{F}}}, \\
K_{\mathrm{R}}=+K \mathrm{e}^{+\mathrm{j} \phi_{\mathrm{B}}}+\frac{2 j \alpha^{2} R_{\mathrm{F}} \Lambda_{\mathrm{T}} \mathrm{e}^{-\mathrm{j} 2 \phi_{\mathrm{T}}}}{3+j \omega C_{\mathrm{F}} R_{\mathrm{F}}}, \\
K_{\mathrm{S}}=+K \mathrm{e}^{-\mathrm{j} \phi_{\mathrm{B}}}+\frac{2 j \alpha^{2} R_{\mathrm{F}} \Lambda_{\mathrm{T}} \mathrm{e}^{+\mathrm{j} 2 \phi_{\mathrm{T}}}}{3+j \omega C_{\mathrm{F}} R_{\mathrm{F}}},
\end{gathered}
$$

In the equations, $\phi_{\mathrm{B}}=\pi / 2$ and $\phi_{\mathrm{T}}=\pi$ are the phase offsets of the grating and the potential, respectively. The coordinates $x=0$ and $x=L$ are located at a distance $\Lambda_{\mathrm{T}} / 4$ away from the centre of the first and last IDT finger (figure 3). The COM parameters in this formulation are the Rayleigh wave velocity of the substrate, $v_{\mathrm{R}}$, the transduction coefficient, $\alpha$, thin film resistance in one transduction period, $R_{\mathrm{F}}$, interdigital capacitance in one transduction period, $C_{\mathrm{F}}$, reflection parameter, $K$, and propagation loss per unit length, $\gamma$. It is worth noting that one transduction period consists of two electrodes in the current case. To facilitate the cascading of uniform transducer elements, solutions of the COM equations can be presented in the $P$ matrix form. In the $P$ matrix representation, the acoustic ports have been treated as scattering ports and the electric port as the admittance port [11]:

$$
\left[\begin{array}{c}
S(0) \\
R(L) \\
I
\end{array}\right]=\left[\begin{array}{lll}
P_{11} & P_{12} & P_{13} \\
P_{21} & P_{22} & P_{23} \\
P_{31} & P_{32} & P_{33}
\end{array}\right]\left[\begin{array}{c}
R_{I}(0) \\
S_{I}(L) \\
V_{0}
\end{array}\right],
$$

where the components of the $P$ matrix are listed in the appendix.

\subsection{COM parameters of a dispersive layered system}

On applying the COM approach to a dispersive layered system, the first encounter problem is the suitability of adopting those COM parameters, which are commonly used in the nondispersive SAW device design. To answer this, we note that the reflection parameter and the change of surface wave velocity generally arise from the mechanical and electrical loading of the IDT electrodes. The contributions from mechanical loading were explained in the literatures $[23,24]$ based on perturbation theory. The theory was developed using the complex reciprocity relation, and is valid for layered waveguide structures as long as the material properties of the waveguide do not vary along the $y$-axis (figure 1). The effect of the electrical loading was derived using the effective permittivity and Floquet's theorem $[25,27]$. The transduction parameter was also derived from the effective permittivity. Therefore, so far as the dispersion of the surface wave is concerned, the formulae for these three COM parameters can be adopted to analyse metal-strip gratings and transducers deposited on a layered substrate.

In this study, the thin film resistance, $R_{\mathrm{F}}$, is assumed to be frequency independent and the propagation loss per unit length, $\gamma$, is adopted from the experimental measurement of [4]. In the following, we employed the matrix formalism of [22] to calculate the frequency dependences of the reflection parameter, the surface wave velocity and the transduction parameter. For purposes of comparison, the design of the IDT/ZnO/sapphire layered SAW filter of [4] has been followed. The aluminium finger electrodes were $100 \mathrm{~nm}$ thick and the finger width to grating period ratio was 0.5 .

3.2.1. Reflection parameter. The reflection parameter $K$ represents the reflectivity of the thin film finger in IDT or the grating. It generally arises from two causes: the electrical loading and the mechanical loading, and can be expressed by $[26,27]$

$$
K=\left[R_{\mathrm{k}}\left(\frac{k_{\mathrm{e}}^{2}(f)}{2}\right)+R_{\mathrm{m}}(f)\left(\frac{h}{\lambda}\right) \sin (\eta \pi)\right] \frac{1}{p},
$$

where $R_{\mathrm{k}}$ and $R_{\mathrm{m}}$ denote the electrical and mechanical parts of the reflection parameter, respectively. $R_{\mathrm{k}}$ is the piezoelectric scattering coefficient, $R_{\mathrm{m}}$ the first-order mechanical scattering coefficient, $h$ the metal film thickness and $\lambda$ the wavelength of the surface wave. $k_{\mathrm{e}}^{2}$ is the electromechanical coupling coefficient and is frequency dependent in this layered structure. $a$ is the finger width and $\eta=a / p$ is the finger width to grating period ratio. The functions $R_{\mathrm{k}}$ and $R_{\mathrm{m}}$ are

$R_{\mathrm{k}}=-\pi s\left[P_{2 \mathrm{~s}}(\cos (\pi \eta))+\frac{P_{\mathrm{S}}(-\cos (\pi \eta)) P_{2 \mathrm{~s}-1}(\cos (\pi \eta))}{P_{-\mathrm{s}}(-\cos (\pi \eta))}\right]$,

$$
\begin{aligned}
R_{\mathrm{m}}= & -\frac{\pi k_{\mathrm{e}}^{2}(f)}{\varepsilon_{\mathrm{S}}(\infty)}\left[\left(\frac{U_{1}(f)}{\varphi(f)}\right)^{2}\left(\alpha_{1}+\rho^{\prime} v_{\mathrm{f}}^{2}\right)\right. \\
& \left.+\left(\frac{U_{2}(f)}{\varphi(f)}\right)^{2}\left(\alpha_{2}+\rho^{\prime} v_{\mathrm{f}}^{2}\right)+\left(\frac{U_{3}(f)}{\varphi(f)}\right)^{2} \rho^{\prime} v_{\mathrm{f}}^{2}\right],
\end{aligned}
$$

where $U_{1}, U_{2}, U_{3}$ and $\varphi$ are the displacements and the surface electrical potential of the surface wave at the surface $z=h_{\mathrm{ZnO}}$ 


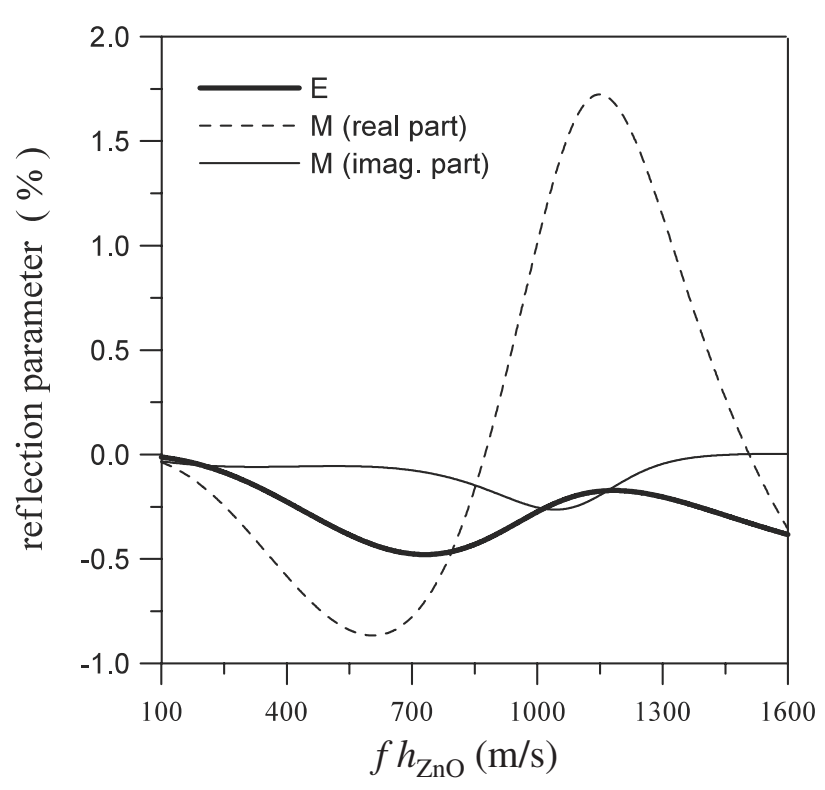

Figure 4. Calculated frequency dependence of the electrical part and the mechanical part of the reflection coefficient.

under the free surface assumption. $\rho^{\prime}, \lambda^{\prime}, \mu^{\prime}$ are the density and Lame constants of the thin film electrode, respectively. The electromechanical coefficient $k_{\mathrm{e}}^{2}$ can be calculated based on the effective permittivity approach [22], while the constants $\alpha_{1}, \alpha_{2}$ are

$$
\alpha_{1}=\frac{4 \mu^{\prime}\left(\lambda+\mu^{\prime}\right)}{\lambda^{\prime}+2 \mu^{\prime}} \quad \text { and } \quad \alpha_{2}=\mu^{\prime} .
$$

In contrast to SAW in the half-space, the displacements, the surface electrical potential and the electromechancial coupling coefficient of the surface wave in the dispersive layered medium are no longer constant; instead, they are frequency dependent. Shown in figure 4 is the calculated frequency dependence of the electrical part (thick solid line) and mechanical part of the reflection coefficient $K$. The thin solid line and the broken line represent the real and imaginary parts of the mechanical reflectivity, respectively. The horizontal axis is the product of the frequency and the thickness of the $\mathrm{ZnO}$ layer $h_{\mathrm{ZnO}}$. Results show that the contribution of the reflection parameter from the electrical part is slightly smaller than that from the mechanical part. The real part of the mechanical part could cancel the electrical part at some frequency. That is, for the cases for which the second-order reflection effects can be ignored, it is possible to get zero reflectivity as long as the metallization ratio and the thickness of the finger electrode are determined appropriately. In figure 5, we show the magnitude (solid line) and the phase (broken line) of the reflection coefficient $K_{\mathrm{R}}$ as a function of the operating frequency for an electrode thickness of $1000 \AA$ and metallization ratio of 0.5 . In this case, zero reflectivity is obtained at a frequency of about $1.5 \mathrm{~km} \mathrm{~s}^{-1}$. It is worth noting that once the reflectivity is equal to zero at the operating frequency, the triple transit echo can be reduced without the split-finger transducers. This results in an increase of the SAW frequency without decreasing the electrode spacing. In addition, from this example, it is clearly seen that the phase of the reflection coefficient is frequency dependent.

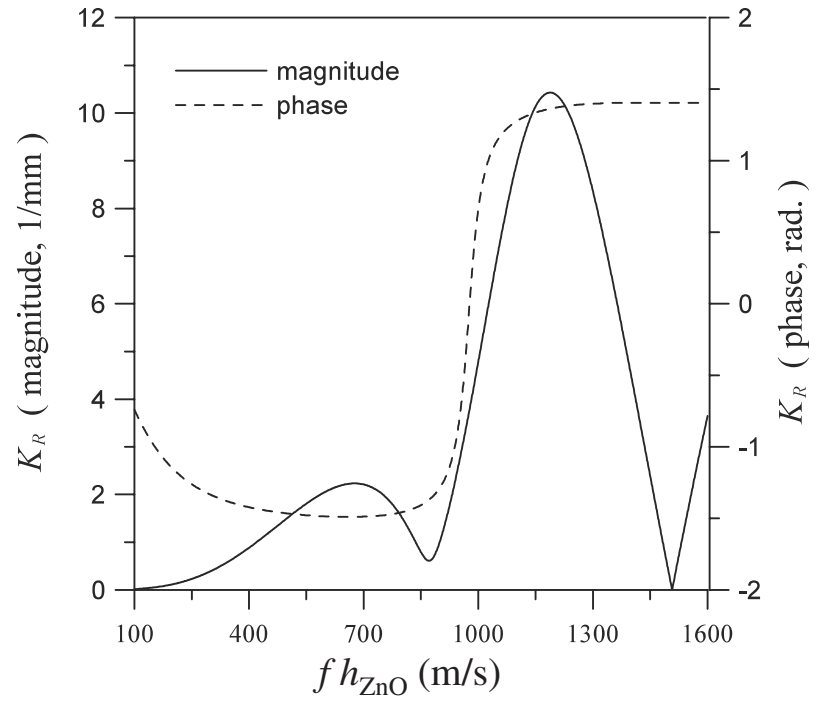

Figure 5. Magnitude and phase of total reflection coefficient as a function of the operating frequency for an electrode thickness of $1000 \AA$.

3.2.2. Surface wave velocity. For small $h / \lambda$, the perturbed surface wave velocity $v_{\mathrm{R}}$ due to short circuit gratings can be approximated as [26, 27]

$v_{\mathrm{R}}=v_{\mathrm{f}}\left(1+\frac{\Delta v}{v_{\mathrm{f}}}\right)=v_{\mathrm{f}}\left(1+D_{\mathrm{k}}\left(\frac{k_{\mathrm{e}}^{2}(f)}{2}\right)+D_{\mathrm{m}}(f)\left(\frac{h}{\lambda}\right)\right)$,

where $v_{\mathrm{f}}$ is the free surface wave velocity of the layered structure with no electrode. $D_{\mathrm{k}}$ and $D_{\mathrm{m}}$ are the changes coming from the electrical and mechanical loadings, respectively. They are given as

$$
D_{\mathrm{k}}=-\pi s\left[1+\frac{P_{\mathrm{S}}(-\cos (\pi \eta))}{P_{-\mathrm{s}}(-\cos (\pi \eta))}\right],
$$

$$
\begin{aligned}
D_{\mathrm{m}}= & \frac{\eta \pi k_{\mathrm{e}}^{2}(f)}{\varepsilon_{\mathrm{S}}(\infty)}\left[\left|\frac{U_{1}(f)}{\varphi(f)}\right|^{2}\left(\alpha_{1}-\rho^{\prime} v_{\mathrm{f}}^{2}\right)\right. \\
& \left.+\left|\frac{U_{2}(f)}{\varphi(f)}\right|^{2}\left(\alpha_{2}-\rho^{\prime} v_{\mathrm{f}}^{2}\right)-\left|\frac{U_{3}(f)}{\varphi(f)}\right|^{2} \rho^{\prime} v_{\mathrm{f}}^{2}\right] .
\end{aligned}
$$

We note that the displacements, the surface electrical potential and the electromechancial coupling coefficient of the surface wave are frequency dependent in a layered SAW structure. In figure 6, we show the calculated frequency dependence of the electrical part (broken line) and the mechanical part (thinner solid lines) of the velocity decrease due to the electrode gratings. The thick solid line is the total velocity decrease as a function of the operating frequency. Results show that the mechanical part of the velocity decrease is larger than the electrical part. The velocity decreases have a maximum of $2.3 \%$ at the normalized frequency of around $1200 \mathrm{~m} \mathrm{~s}^{-1}$.

3.2.3. Transduction parameter. The transduction coefficient, $\alpha$, which is responsible for the excitation efficiency of the IDT, can be derived as [28]

$$
\alpha(\omega)=\frac{Q_{\mathrm{F}}(\beta)}{\Lambda_{\mathrm{T}}} \sqrt{\frac{\omega W \Gamma_{\mathrm{S}}}{2}}
$$




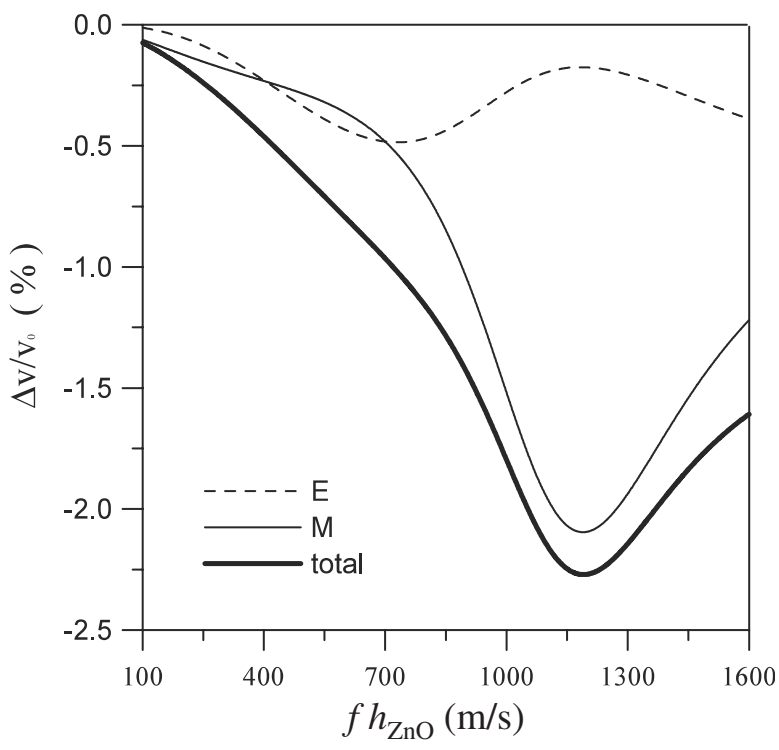

Figure 6. Calculated frequency dependence of the electrical part and the mechanical part and total velocity decrease due to the electrode gratings.

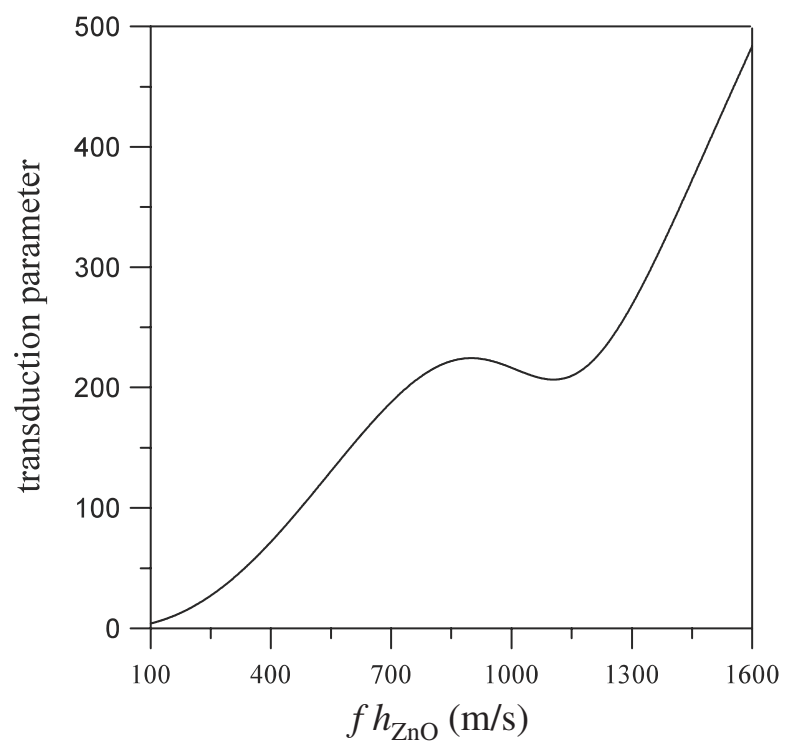

Figure 7. Calculated frequency dependence of the transduction coefficient.

with the Fourier transform of the elemental charge density, $Q_{\mathrm{F}}$, defined as

$$
\begin{gathered}
Q_{\mathrm{F}}(\beta)=\varepsilon_{\mathrm{S}}(\infty) \frac{2 \sin (\pi s)}{P_{-\mathrm{S}}(-\cos \eta)} P_{\mathrm{m}}(\cos \eta) \\
\text { for } m \leqslant \frac{\beta p}{2 \pi} \leqslant m+1,
\end{gathered}
$$

where $W$ is the aperture of the IDT, $\varepsilon_{\mathrm{S}}(\infty)$ is the surface effective permittivity as a function of the slowness, $m$ is an integer, $s=(\beta p / 2 \pi)-m, P_{-\mathrm{s}}(-\cos \eta)$ is a Legendre function and $P_{\mathrm{m}}(\cos \eta)$ is a Legendre polynomial. The definition of the coefficient $\Gamma_{\mathrm{S}}$ can be found in $[22,29]$. Owing to the constant $\Gamma_{\mathrm{S}}$, the transduction coefficient in the half-space SAW is proportional to the square root of the frequency. In figure 7 , the calculated frequency dependence of the transduction coefficient is shown. It shows that the transduction coefficient

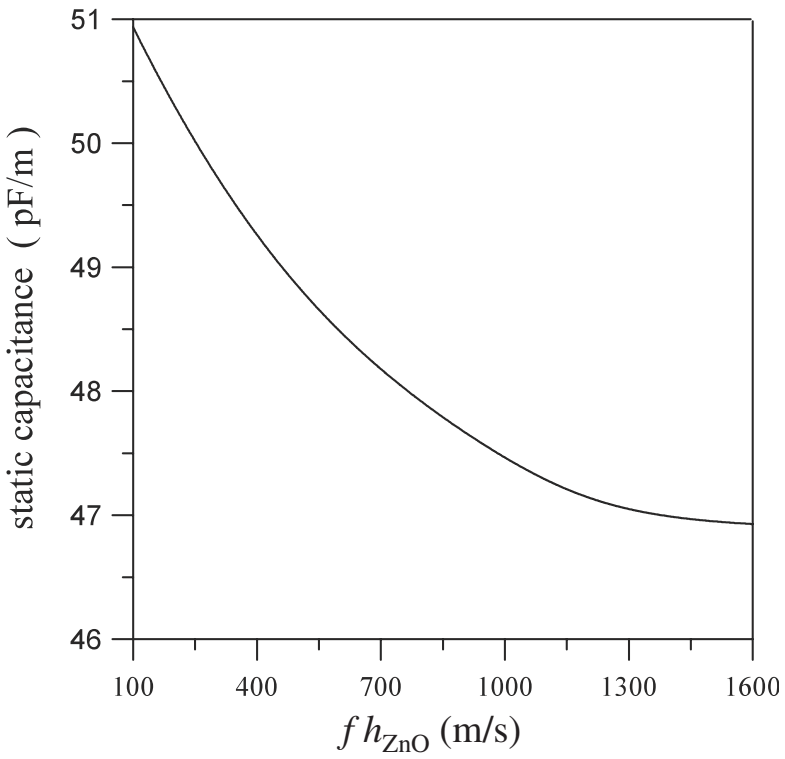

Figure 8. Calculated frequency dependence of the static capacitance.

in the dispersive layered SAW filter is not simply proportional to the square root of frequency due to the dispersion of $\Gamma_{S}$.

3.2.4. Thin film finger capacitance. When thin electrodes are deposited between vacuum and a layered half-space substrate, the finger capacitance per metre of finger length for a strip of width $p$ can be expressed empirically as [30]

$C_{\mathrm{S}}\left(\frac{\mathrm{pF}}{\mathrm{m}}\right)=k\left(\varepsilon_{\mathrm{S}}+1\right)+k\left(\varepsilon_{1}-\varepsilon_{\mathrm{S}}\right)\left[1-\exp \left(-4.6 \frac{h_{\mathrm{ZnO}}}{p}\right)\right]$,

where the empirical expression of $k$ is

$$
k=6.5\left(\frac{a}{p}\right)^{2}+1.08\left(\frac{a}{p}\right)+2.37,
$$

$\varepsilon_{\mathrm{S}}$ is the dielectric constant of the $R$-plane sapphire substrate, and $\varepsilon_{1}$ the dielectric constant of the $\mathrm{ZnO}$ film, i.e.

$$
\varepsilon_{1}=\left(\varepsilon_{11} \varepsilon_{33}-\varepsilon_{13}^{2}\right)^{1 / 2} .
$$

The finger capacitance formula is valid for the ranges $0.2<$ $a / p<0.8$ and $0.1<\varepsilon_{\mathrm{S}}<50$. In figure 8 , we show the calculated frequency dependence of the finger capacitance. In contrast to the half-space, the finger capacitance in the dispersive layered SAW filter is no longer constant; instead, it is frequency dependent. The finger capacitance decreases with increasing $f h_{\mathrm{ZnO}}$.

3.2.5. Thin film finger resistance. For a single electrode type IDT, the thin film finger resistance in one transduction period can be determined by [28]

$$
R_{\mathrm{F}}=\frac{2 \rho W}{3 a}
$$

where $\rho$ is the sheet resistance of the metal film. For an aluminium thin film with thickness $h(\mu \mathrm{m})$ lying between 0.05 and $0.3 \mu \mathrm{m}$, the sheet resistance can be approximated as

$$
\rho \approx \frac{0.04}{h} \quad(\Omega \text { per square }) .
$$


3.2.6. Propagation loss $\gamma$. Unlike the Rayleigh wave propagation in single-crystal material, the propagation loss of SAWs in the sputtered $\mathrm{ZnO}$ caused by the scattering loss due to inhomogeneity has to be considered. Therefore, to compare the simulated and experimental responses of a layered SAW filter, we employed the experimental propagation loss of the $\mathrm{ZnO} / \mathrm{R}$ plane sapphire layered system reported in [4]. The propagation loss reported in [4] is around $10 \mathrm{~dB} \mathrm{~cm}^{-1}$ at the frequency of $440 \mathrm{MHz}$. In this paper, the frequency range of interest is small, so we assumed that the variation of propagation loss is independent of the frequency.

\section{Insertion loss of a two-port IDT/ZnO/sapphire layered SAW filter}

In this section, a two-port IDT/ZnO/sapphire layered SAW filter is analysed based on the modified COM model described in sections 2 and 3 . In order to compare with the existing experimental data, we follow all the design parameters utilized in [4]. The thickness of the $\mathrm{ZnO}$ layer is $h_{\mathrm{ZnO}}=1.45 \mu \mathrm{m}$, the design frequency is $440 \mathrm{MHz}$. The free surface velocity of the 0th Rayleigh mode at $440 \mathrm{MHz}$ is calculated as $v_{\mathrm{f}}=$ $5007 \mathrm{~m} \mathrm{~s}^{-1}$, and the coupling coefficient as $1.1 \%$. The transduction period of the IDT is $\Lambda_{\mathrm{T}}=11.2 \mu \mathrm{m}$ and the number of IDT pairs is 37 . The aperture of the SAW is $W=750 \mu \mathrm{m}$, the metalization ratio $\eta=0.5$ and the thickness of the finger electrode is $1000 \AA$. The propagation length is $3.4 \mathrm{~mm}$. In figure 9, we show the simulated insertion loss curves of two-port IDT/ZnO/sapphire layered SAW filters with and without considering dispersion. Results showed that the null frequency bandwidth of the one calculated including the dispersion effect is narrower than that obtained without taking into account the dispersion.

The solid line shown in figure 10 corresponds to the insertion loss measured in [4] and the broken line is the calculated insertion loss based on this proposed model. Results show that the proposed model could predict the bandwidth and most of the frequency characteristics of the layered SAW filter with quite a good accuracy. However, the calculated insertion loss is slightly smaller than the measured one. The mismatch

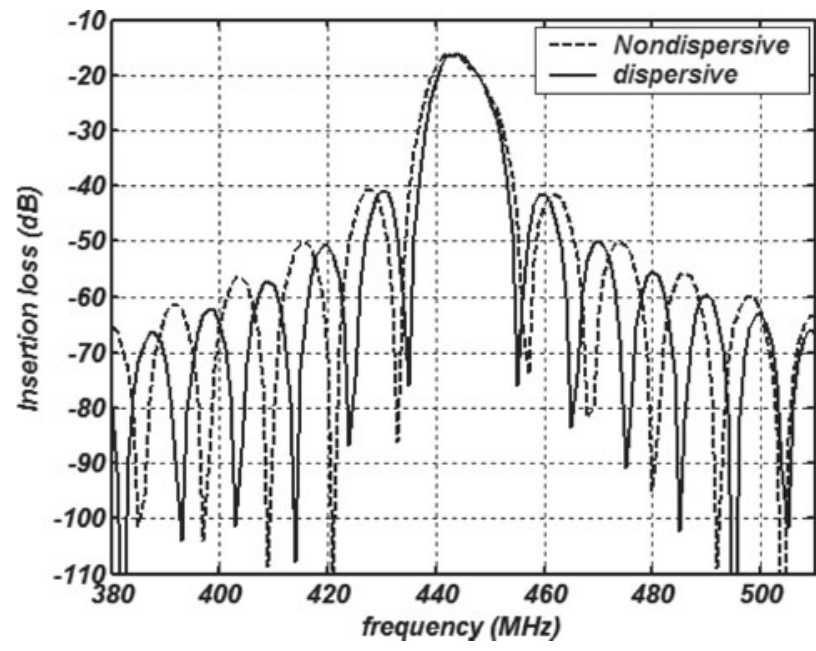

Figure 9. Calculated insertion loss based on the proposed model with and without considering dispersion. of the insertion loss may be due to the over prediction of the piezoelectric properties of the deposited $\mathrm{ZnO}$ film. Therefore, instead of using the calculated electromechanical coupling coefficient, we tried the measured values of [4], i.e. $k_{\mathrm{e}}^{2}=0.9 \%$, to calculate the frequency response of the layered SAW filter as shown in figure 11. Results have shown that the calculated insertion loss matched the measured one quite well.

\section{Conclusion}

In this paper, we proposed a modified COM approach that includes the SAW dispersion effects to calculate the frequency response of a layered SAW filter. The effective permittivity approach was used to calculate the frequency dependences of the electromechanical coupling coefficient, displacements and electrical potential of surface waves in the layered system. The frequency dependences of the COM parameters have been calculated for the first time and the result shows that the dispersive effect has to be considered in the design. The simulated insertion loss of a two-port IDT/ZnO/sapphire

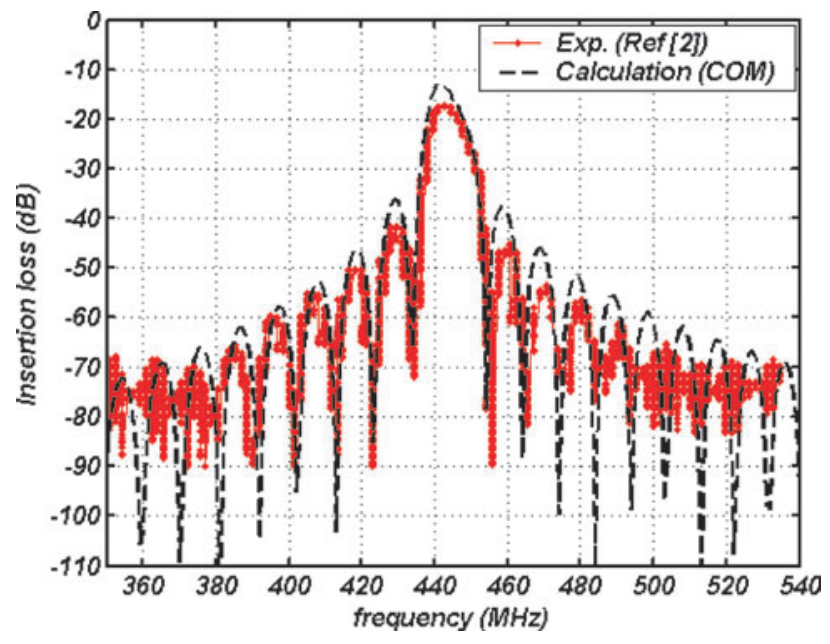

Figure 10. Measured insertion loss, from [4] (- - ), and the calculated insertion loss based on the proposed model $(\cdots \cdots)$.

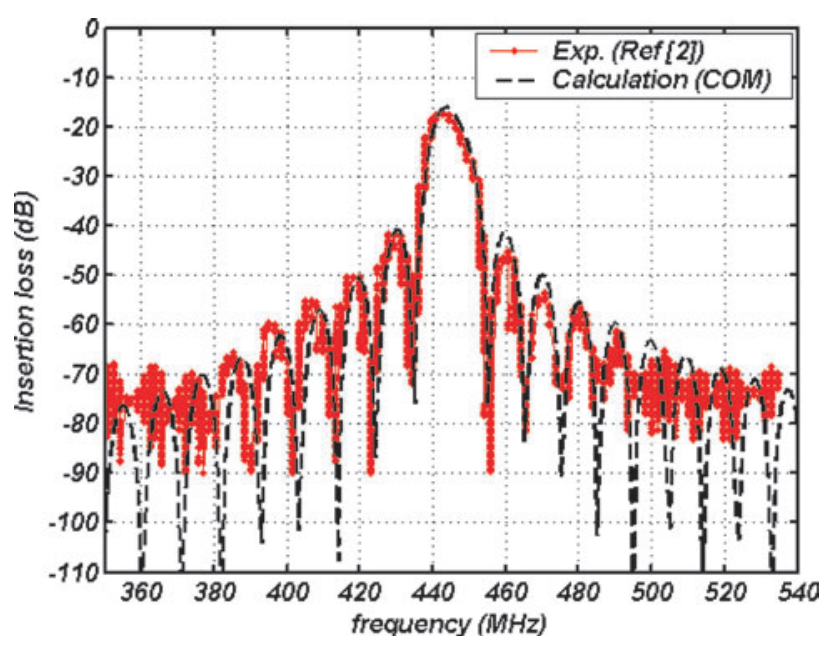

Figure 11. Measured insertion loss, from [4] (- - ), and the calculated insertion loss based on the proposed model with the measured $k_{\mathrm{e}}^{2}=0.9 \%(\cdots \cdots)$. 
layered SAW filter is compared with that of the measured one in [4]. Results have shown that except for a slight deviation of the insertion loss, the proposed approach gave a satisfactory prediction of the frequency response of the layered SAW filter. We note that the current results were based on the assumption of a first-order mechanical reflection; therefore, the efficiency of the proposed model for a highly reflective grating design awaits further investigation.

\section{Acknowledgments}

The authors thank the National Science Council of Taiwan for the financial support for this research, through the grant NSC90-2212-E-002-156.

\section{Appendix}

The components of the $P$-matrix are [11]

$$
\begin{aligned}
P_{11}= & \frac{+j K_{\mathrm{S}} \sin (D L)}{D \cos (D L)+j \Delta \sin (D L)}, \\
P_{12}= & \frac{D}{D \cos (D L)+j \Delta \sin (D L)} \mathrm{e}^{-\mathrm{j} k_{0} L}, \\
P_{13}= & +j L\left(\frac{\sin (D L / 2)}{D L / 2}\right) \\
& \times\left[\frac{\alpha_{\mathrm{S}} D \cos (D L / 2)+j\left(K_{\mathrm{S}} \alpha_{\mathrm{R}}+\Delta \alpha_{\mathrm{S}}\right) \sin (D L / 2)}{D \cos (D L)+j \Delta \sin (D L)}\right],
\end{aligned}
$$

$$
\begin{aligned}
P_{22}= & \frac{+j K_{\mathrm{R}} \sin (D L)}{D \cos (D L)+j \Delta \sin (D L)} \mathrm{e}^{-\mathrm{j} 2 k_{0} L}, \\
P_{23}= & +j L\left(\frac{\sin (D L / 2)}{D L / 2}\right) \\
& \times\left[\frac{\alpha_{\mathrm{R}} D \cos (D L / 2)+j\left(K_{\mathrm{R}} \alpha_{\mathrm{S}}+\Delta \alpha_{\mathrm{R}}\right) \sin (D L / 2)}{D \cos (D L)+j \Delta \sin (D L)}\right] \\
& \times \mathrm{e}^{-\mathrm{j} k_{0} L}, \\
P_{33}= & -j 2\left(\frac{K_{\mathrm{S}} \alpha_{\mathrm{R}}^{2}+K_{\mathrm{R}} \alpha_{\mathrm{S}}^{2}+2 \Delta \alpha_{\mathrm{S}} \alpha_{\mathrm{R}}}{D^{3}}\right) \\
& \times\left[D L-\frac{D \sin (D L)+j \Delta(1-\cos (D L))}{D \cos (D L)+j \Delta \sin (D L)}\right] \\
& -2\left(\frac{\Delta\left(K_{\mathrm{S}} \alpha_{\mathrm{R}}^{2}+K_{\mathrm{R}} \alpha_{\mathrm{S}}^{2}\right)+2 K_{\mathrm{R}} K_{\mathrm{S}} \alpha_{\mathrm{R}} \alpha_{\mathrm{S}}}{D^{3}}\right) \\
& \times\left(\frac{1-\cos (D L)}{D \cos (D L)+j \Delta \sin (D L)}\right) \\
+ & j\left(\frac{3 \omega C_{\mathrm{F}} L / \Lambda_{\mathrm{T}}}{3+j \omega R_{\mathrm{F}} C_{\mathrm{F}}}\right),
\end{aligned}
$$

where $\Delta=k_{\mathrm{E}}-k_{0}$ and $D=\sqrt{\Delta^{2}-K_{\mathrm{R}} K_{\mathrm{S}}}$.

\section{References}

[1] Kino G S and Wagers R S 1973 Theory of interdigital couplers on nonpiezoelectric substrates J. Appl. Phys. 44 1480-8
[2] Adler E L and Solie L $1995 \mathrm{ZnO}$ on diamond: SAWs and pseudo-SAWs Proc. IEEE Ultrason. Symp. pp 341-4

[3] Nakahata H, Hachigo A, Higaki K, Fujii S, Shikata S and Fujimori N 1995 Theoretical study on SAW characteristics of layered structures including a diamond layer IEEE UFFC 42 362-75

[4] Mitsuyu T, Ono S and Wasa K 1980 Structures and SAW properties of rf-sputtered single-crystal films of $\mathrm{ZnO}$ on sapphire J. Appl. Phys. 51 2464-70

[5] Nakahata H, Higaki K S, Fujii S, Hachigo A, Kitabayashi H, Tanabe K, Seki Y and Shikata S 1995 SAW devices on diamond IEEE Ultrason. Symp. pp 361-70

[6] Emanetoglu N W, Liang S, Gorla C and Lu Y 1997 Epitaxial growth and characterization of high quality $\mathrm{ZnO}$ films for surface acoustic wave applications IEEE Ultrason. Symp. pp 195-9

[7] Nakahata H, Higaki K, Hachigo A, Fujii S, Uemura T and Shikata S 1998 Diamond SAW filter for $2.488 \mathrm{Gbps}$ retiming IEEE Ultrason. Symp. pp 319-22

[8] Cross P S and Schmidt R V 1997 Coupled acoustic wave resonators Bell Syst. Technol. J. 56 1447-82

[9] Wright P V 1981 A coupling-of-mode analysis of SAW grating structures $P h D$ Thesis Department of Electrical Engineering, Cambridge, MA, USA: MIT

[10] Chen D P and Haus H A 1985 Analysis of metal strip SAW gratings and transducers IEEE Trans. Son. Ultrason. SU-32 395-408

[11] Abbott B P 1989 A coupling-of-modes model for SAW transducers with arbitrary reflectivity weighting $P h D$ Thesis Department of Electrical Engineering, University of Central Florida Orlando, Florida, USA

[12] Wright P V 1989 A new generalized modeling of SAW transducers and gratings Proc. 43rd Ann. Fre. Contr. Symp. pp 596-605

[13] Wu T-T, Wang S-M, Chen Y-Y, Wu T-Y, Chang P-Z, Huang L-S, Wang C-L, Wu C-W and Lee C-K 2002 Inverse determination of coupling of modes parameters of surface acoustic wave resonators Japan. J. Appl. Phys. 41 $6610-5$

[14] Endoh G, Hashimoto K and Yamaguchi M 1995 Surface acoustic wave propagation characterisation by finite element method and spectral domain analysis Japan. $J$. Appl. Phys. 34 2638-41

[15] Ventura P, Hode J M and Solal M A 1995 A new efficient combined FEM and periodic Green's function formalism for the analysis of periodic SAW structures IEEE Ultrason. Symp. pp 263-8

[16] Koskela J, Plessky V P and Salomaa M 1999 SAW/LSAW COM parameter extraction from computer experiments with harmonic admittance of a periodic array of electrodes IEEE UFFC 46 806-16

[17] Emanetoglu N W, Patounakis G, Liang S, Gorla C R, Wittstruck R and Lu Y 2001 Analysis and SAW properties

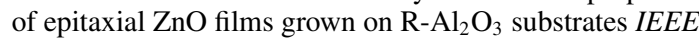
UFFC 48 1389-94

[18] Smith P M 2001 Dyadic Green's functions for multi-layer SAW substrates IEEE UFFC 48 171-9

[19] Hachigo A and Malocha D C 1998 SAW device modeling including velocity dispersion based on $\mathrm{ZnO} /$ diamond/ $\mathrm{Si}$ layered structures IEEE UFFC $\mathbf{4 5}$ 660-5

[20] Gryba T, Haddou A, Sadaune V, Zhang V, Lefebvre J E, Doghech E, Cattan E and Remiens D 2001 Integration of rf filters on GaAs substrate Proc. IEEE Ultrason. Symp. pp 57-60

[21] Abe H and Sato T 2000 Boundary integral equentionsa from hamilton's principle for surface acoustic waves under periodic metal gratings IEEE UFFC 47 1601-3

[22] Wu T-T and Chen Y-Y 2002 Exact analysis of dispersive SAW devices on $\mathrm{ZnO} /$ diamond/Si layered structures IEEE UFFC 49 142-9

[23] Auld B A 1990 Acoustic Fields and Waves in Solids (Florida: Krieger) 
[24] Datta S and Hunsinger B J 1979 First-order reflection coefficient of surface acoustic waves from thin-strip overlays J. Appl. Phys. 50 5661-5

[25] Datta S and Hunsinger B J 1980 An analytical theory for the scattering of surface acoustic waves by a single electrode in a periodic array on a piezoelectric substrate J. Appl. Phys. 51 4817-23

[26] Thorvaldsson T 1989 Analysis of the natural single phase unidirectional SAW transducer IEEE Ultrason. Symp. pp 91-6
[27] Abbott B P 1991 A derivation of the coupling-of-modes parameters based on the scattering analysis of SAW transducers and gratings IEEE Ultrason. Symp. pp 5-10

[28] Morgan D P 1991 Surface-Wave Devices for Signal Processing (New York: Elsevier)

[29] Matthews H 1977 Surface Wave Filters: Design, Construction and Use equation (2.30) (New York: Wiley) p 66

[30] Farnell G W, Cermak I A, Silvester P and Wong S K 1970 Capacitance and field distributions for interdigital surfacewave transducer IEEE Trans. Son. Ultrason. 17 188-95 